OPEN ACCESS

Edited by:

Nianyin Zeng,

Xiamen University, China

Reviewed by:

Liansheng Wang,

Xiamen University, China

Chen Yang,

Hangzhou Dianzi University, China

*Correspondence:

Chaosheng Tang

tcs@hpu.edu.cn

Junding Sun

sunjd@hpu.edu.cn

Yu-Dong Zhang

yudongzhang@ieee.org

†These authors have contributed equally to this work

Specialty section:

This article was submitted to

Neurodegeneration,

a section of the journal

Frontiers in Psychiatry

Received: 14 February 2019

Accepted: 21 March 2019

Published: 11 April 2019

Citation:

Wang S-H, Xie S, Chen X, Guttery DS, Tang C, Sun J and Zhang Y-D (2019)

Alcoholism Identification Based on an AlexNet Transfer Learning Model.

Front. Psychiatry 10:205.

doi: 10.3389/fpsyt.2019.00205

\section{Alcoholism Identification Based on an AlexNet Transfer Learning Model}

\author{
Shui-Hua Wang ${ }^{1,2,3+}$, Shipeng Xie ${ }^{4 \dagger}$, Xianqing Chen ${ }^{5 t}$, David S. Guttery ${ }^{3+}$, \\ Chaosheng Tang ${ }^{1 *}$, Junding Sun ${ }^{1 *}$ and Yu-Dong Zhang ${ }^{1,3,6 * t}$ \\ ${ }^{1}$ School of Computer Science and Technology, Henan Polytechnic University, Jiaozuo, China, ${ }^{2}$ School of Architecture \\ Building and Civil Engineering, Loughborough University, Loughborough, United Kingdom, ${ }^{3}$ Department of Informatics, \\ University of Leicester, Leicester, United Kingdom, ${ }^{4}$ College of Telecommunications and Information Engineering, Nanjing \\ University of Posts and Telecommunications, Nanjing, China, ${ }^{5}$ Department of Electrical Engineering, College of Engineering, \\ Zhejiang Normal University, Jinhua, China, ${ }^{6}$ Guangxi Key Laboratory of Manufacturing System and Advanced Manufacturing \\ Technology, Guilin, China
}

Aim: This paper proposes a novel alcoholism identification approach that can assist radiologists in patient diagnosis.

Method: AlexNet was used as the basic transfer learning model. The global learning rate was small, at $10^{-4}$, and the iteration epoch number was at 10 . The learning rate factor of replaced layers was 10 times larger than that of the transferred layers. We tested five different replacement configurations of transfer learning.

Results: The experiment shows that the best performance was achieved by replacing the final fully connected layer. Our method yielded a sensitivity of $97.44 \% \pm 1.15 \%$, a specificity of $97.41 \pm 1.51 \%$, a precision of $97.34 \pm 1.49 \%$, an accuracy of $97.42 \pm$ $0.95 \%$, and an F1 score of $97.37 \pm 0.97 \%$ on the test set.

Conclusion: This method can assist radiologists in their routine alcoholism screening of brain magnetic resonance images.

\footnotetext{
Keywords: alcoholism, transfer learning, AlexNet, data augmentation, convolutional neural network, dropout, local response normalization, magnetic resonance imaging
}

\section{INTRODUCTION}

Alcoholism (1) was previously composed of two types: alcohol abuse and alcohol dependence. According to current terminology, alcoholism differs from "harmful drinking" (2), which is an occasional pattern of drinking that contributes to increasing levels of alcohol-related ill-health. Today, it is defined depending on more than one of the following conditions: alcohol is strongly desired, usage results in social problems, drinking large amounts over a long time period, difficulty in reducing alcohol consumption, and usage resulting in non-fulfillment of everyday responsibilities.

Alcoholism affects all parts of the body, but it particularly affects the brain. The size of gray matter and white matter of alcoholism subjects are less than age-matched controls (3), and this shrinkage can be observed using magnetic resonance imaging (MRI). However, neuroradiological diagnosis using MR images is a laborious process, and it is difficult to detect minor alterations in the brain of alcoholic patient. Therefore, development of a computer vision-based automatic smart alcoholism identification program is highly desirable to assist doctors in making a diagnosis.

Within the last decade, studies have developed several promising alcoholism detection methods. Hou (4) put forward a novel algorithm called predator-prey adaptive-inertia chaotic particle swarm 
optimization (PAC-PSO), and applied it to identify alcoholism in MR brain images. Lima (5) proposed to use Haar wavelet transform (HWT) to extract features from brain images, and the authors used HWT to detect alcoholic patients. Macdonald (6) developed a logistic regression (LR) system. Qian (7) employed the cat swarm optimization (CSO) and obtained excellent results in the diagnosis of alcoholism. Han (8) used wavelet Renyi entropy (WRE) to generate a new biomarker; whereas Chen (9) used a support vector machine, which was trained using a genetic algorithm (SVM-GA) approach. Jenitta and Ravindran (10) proposed a local mesh vector co-occurrence pattern ( $\mathrm{LMCoP})$ feature for assisting diagnosis.

Recently, deep learning has attracted attention in many computer vision fields, e.g., synthesizing visual speech (11), liver cancer detection (12), brain abnormality detection (13), etc. As a result, studies are now focused on using deep learning techniques for alcoholism detection. Compared to manual feature extraction methods (14-18), deep learning can "learn" the features of alcoholism. For example, Lv (19) established a deep convolutional neural network (CNN) containing seven layers. Their experiments found that their model obtained promising results, and the stochastic pooling provided better performance than max pooling and average pooling. Moreover, Sangaiah (20) developed a ten-layer deep artificial neural network (i.e., three fully-connected layers and seven conv layers), which integrated advanced techniques, such as dropout and batch normalization, into their neural network.

Transfer learning (TL) is a new pattern recognition problemsolver (21-23). TL attempts to transfer knowledge learned using one or more source tasks (e.g., ImageNet dataset) and uses it to improve learning in a related target task (24). In perspective of realistic implementation, the advantages of TL compared to plain deep learning are: (i) TL uses a pretrained model as a starting point; (ii) fine-tuning a pretrained model is usually easier and faster than training a randomly-initialized deep neural network.

The contribution of this paper is that we may be the first to apply transfer learning in this field of alcoholism identification. We used AlexNet as the basic transfer learning model and tested different transfer configurations. Further, the experiments showed that the performance (sensitivity, specificity, precision, accuracy, and F1 score) of our model is $>97 \%$, which is superior to state-of-the-art approaches. We also validated the effectiveness of using data augmentation which further improves the performance of our model.

\section{DATA PREPROCESSING}

\section{Datasets}

This study was approved by the ethical committee of Henan Polytechnic University. Three hundred seventy-nine slices were obtained in which there are 188 alcoholic brain images and 191 non-alcoholic brain images. We divided the dataset into three parts: a training set containing 80 alcoholic brain images and 80 non-alcoholic brain images; A validation set containing 30 alcoholic brain images and 30 non-alcoholic brain images; a test set containing 78 alcoholic brain images and 81 non-alcoholic brain images. The division is shown in Table $\mathbf{1 .}$
TABLE 1 | Dataset division into training, validation, and test sets.

\begin{tabular}{lccc}
\hline & Alcoholic & Non-alcoholic & Total \\
\hline Training & 80 & 80 & 160 \\
Validation & 30 & 30 & 60 \\
Test & 78 & 81 & 159 \\
Total & 188 & 191 & 379 \\
\hline
\end{tabular}

TABLE 2 | Data augmentation.

\begin{tabular}{lccc}
\hline & Alcoholic & Non-alcoholic & Total \\
\hline Original Image & 80 & 80 & 160 \\
DA_I: Noise Injection & 2,400 & 2,400 & 4,800 \\
DA_II: Scaling & 2,400 & 2,400 & 4,800 \\
DA_III: Random Translation & 2,400 & 2,400 & 4,800 \\
DA_IV: Image Rotation & 2,400 & 2,400 & 4,800 \\
DA_V: Gamma Correction & 2,400 & 2,400 & 4,800 \\
Horizontal-flipped Image & 80 & 80 & 160 \\
DA_I: Noise Injection & 2,400 & 2,400 & 4,800 \\
DA_II: Scaling & 2,400 & 2,400 & 4,800 \\
DA_III: Random Translation & 2,400 & 2,400 & 4,800 \\
DA_IV: Image Rotation & 2,400 & 2,400 & 4,800 \\
DA_V: Gamma Correction & 2,400 & 2,400 & 4,800 \\
New Training Data & 24,160 & 24,160 & 48,320
\end{tabular}

\section{Data Augmentation}

To improve the performance of deep learning, data augmentation (DA) (25) was introduced. This was done because our deep neural network model has many parameters, so we needed to show that our model contains a proportional amount of sample images to achieve optimal performance. For each original image, we generated a horizontally flipped image. Then, for both original and horizontal-flipped images, we applied the following five DA techniques: (i) noise injection, (ii) scaling, (iii) random translation, (iv) image rotation, and (v) gamma correction. Each of those methods produced 30 new images.

Gaussian noise with zero-mean and variance of 0.01 was applied to every image. Scaling was used with a scaling factor of $0.7-1.3$, with an increase of 0.02 . Random translation was utilized with a random shift within $[-4040]$ pixels. Image rotation with rotation angle varies from $-30^{\circ}$ to $30^{\circ}$ and a step of $2^{\circ}$ was employed. Gamma correction with gamma value varies from 0.4 to 1.6 with a step of 0.04 was utilized.

The DA result is shown in Table 2. Each image generated $\left(1+30^{*} 5\right)^{*} 2=302$ images including itself. After DA, the training set had 24,160 alcoholism brain images and 24,160 healthy brain images. Altogether, the new training set consisted of a balanced $160 * 320=48,320$ samples.

\section{METHODOLOGY}

\section{Fundamentals of Transfer Learning}

The core knowledge of transfer learning (TL) is shown in Figure 1. The core is to use a relatively complex and successful pre-trained model, trained from a large data source, e.g., 


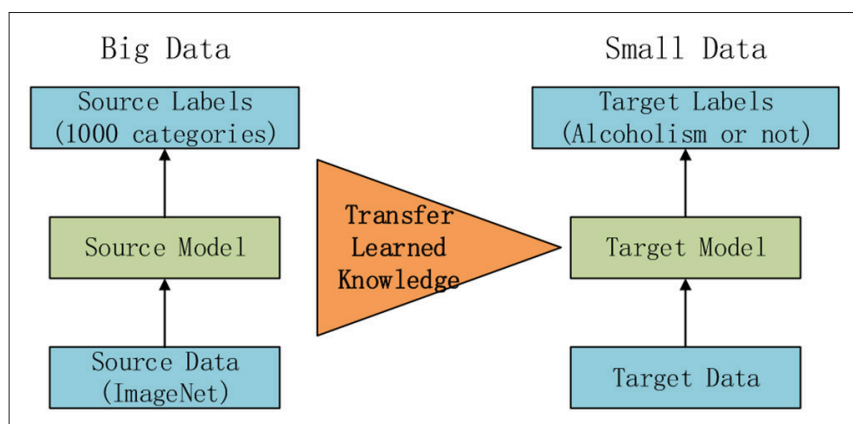

FIGURE 1 | Idea of transfer learning.

ImageNet, which is the large visual database developed for visual object recognition research (26). It contains more than $14,000,000$ hand-annotated images and at least one million images are provided with bounding boxes. ImageNet contains more than 20,000 categories (27). Usually, pretrained models are trained on a subset of ImageNet with 1,000 categories. Then we "transferred" the learnt knowledge to the relatively simplified tasks (e.g., classifying alcoholism and non-alcoholism in this study) with a small amount of private data.

Two attributes are important to help the transfer (28): (i) The success of the pretrained model can promote the exclusion of user intervention with the boring hyper-parameter tuning of new tasks; (ii) The early layers in pretrained models can be determined as feature extractors that help to extract low-level features, such as edges, tints, shades, and textures.

Traditional TL only retrains the new layers (29). In this study, we initially used the pretrained model, and then re-trained the whole structure of the neural network. Importantly, the global learning rate is fixed, and the transferred layers will have a low factor, while newly-added layers will have a high factor.

\section{AlexNet}

AlexNet competed in the ImageNet challenge (30) in 2012, achieved a top- 5 error of only $15.3 \%$, more than $10.8 \%$ better than the result of the runner-up that used the shallow neural network. Original AlexNet was performed on two graphical processing units (GPUs). Nowadays, researchers tend to use only one GPU to implement AlexNet. Figure 2 illustrates the structure of AlexNet. This study only counts layers associated with learnable weights. Hence, AlexNet contains five conv layers (CL) and three fullyconnected layers (FCL), totaling eight layers.

The details of learnable weights and biases of AlexNet are shown in Table 3. The total weights and biases of AlexNet are $60,954,656+10,568=60,965,224$. In Matlab, the variable is stored in single-float type, taking four bytes for each variable. Hence, in total we needed to allocate $233 \mathrm{MB}$.

\section{Common Layers in AlexNet}

Compared to traditional neural networks, there are several advanced techniques used in AlexNet. First, CLs contain a set of learnable filters. For example, the user has a $3 \mathrm{D}$ input with a size of $P_{W} \times P_{H} \times D$, a $3 \mathrm{D}$ filter with a size of $Q_{W} \times Q_{H} \times D$. As a consequence, the size of the output activation map is $S_{W} \times S_{H}$. The value of $S_{W}$ and $S_{H}$ can be obtained by

$$
\begin{array}{r}
S_{W}=1+\frac{P_{W}-Q_{W}+2 \beta}{\mu} \\
S_{H}=1+\frac{P_{H}-Q_{H}+2 \beta}{\mu}
\end{array}
$$

where $\mu$ is the stride size and $\beta$ represents the margin. Commonly, there may be $T$ filters. One filter will generate one 2D feature map, and $T$ filters will yield an activation map with a size of $S_{W} \times S_{H} \times T$. An illustration of convolutional procedure is shown in Figure 3. The "feature learning" in the filters here, can be regarded as a replacement of the "feature extraction" in traditional machine learning.

Second, the rectified linear unit (ReLU) function was employed to replace the traditional sigmoid function $S(x)$ in terms of the activation function (31). The reason is because the sigmoid function may come across a gradient vanishing problem in deep neural network models.

$$
S(x)=\frac{1}{1+\exp (-x)}
$$

Therefore, the ReLU was proposed and defined as follows:

$$
\operatorname{Re} \operatorname{LU}(x)=\max (0, x)
$$

The gradient of ReLU is one at all times, when the input is larger than or equal to zero. Scholars have proven that the convergence speed of deep neural networks, with ReLU as the activation function, is $6 \mathrm{x}$ quicker than traditional activation functions. Therefore, the new ReLU function greatly accelerates the training procedure.

Third, a pooling operation is implemented with two advantages: (i) It can reduce the size of the feature map, and thus reduce the computation burden. (ii) It ensures that the representation becomes invariant to the small translation of the input. Map pooling (MP) is a common technique that chooses the maximum value among a $2 \times 2$ region of interest. Figure 4 shows a toy example of MP, with a stride of 2 and kernel size of 2 .

The fourth improvement is the "local response normalization (LRN).” Krizhevsky et al. (26) proposed the LRNs in order to aid generalization. Suppose that $a_{i}$ represents a neuron computed by applying kernel $i$ and ReLU non-linearity, then the responsenormalized neuron $b_{i}$ will be expressed as:

$$
b_{i}=\frac{a_{i}}{\left(m+\alpha \sum_{s=\max (0, i-z / 2)}^{\min (Z-1, i+z / 2)} a_{s}^{2}\right)^{\beta}}
$$

where $z$ is the window channel size, controlling the number of channels used for normalization of each element, and $Z$ is the gross number of kernels in that layer. Hyperparameters are set as: $\beta=0.75, \alpha=10^{-4}, m=1$, and $z=5$. 


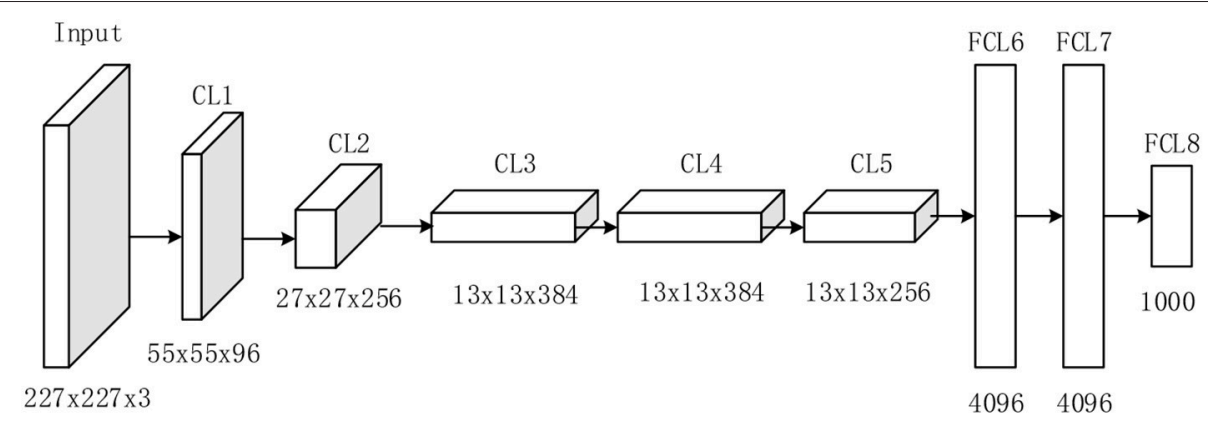

FIGURE 2 | Structure of AlexNet (5 CLs and 3 FCLs).

TABLE 3 | Learnable layers in AlexNet.

\begin{tabular}{|c|c|c|}
\hline Name & Weights & Biases \\
\hline CL1 & $11^{\star} 11^{\star} 3^{\star} 96=34,848$ & $1^{\star} 1^{\star} 96=96$ \\
\hline CL2 & $5^{\star} 5^{\star} 48^{\star} 256=307,200$ & $1^{*} 1 * 256=256$ \\
\hline CL3 & $3^{\star} 3^{\star} 256^{\star} 384=884,736$ & $1 * 1 * 384=384$ \\
\hline CL4 & $3^{\star} 3^{\star} 192^{\star} 384=663,552$ & $1 * 1 * 384=384$ \\
\hline CL5 & $3^{\star} 3^{\star} 192^{\star} 256=442,368$ & $1^{*} 1 * 256=256$ \\
\hline FCL6 & $4096 * 9216=37,748,736$ & $4096^{*} 1=4,096$ \\
\hline FCL7 & $4096 * 4096=16,777,216$ & $4096^{*} 1=4,096$ \\
\hline FCL8 & $1000 * 4096=4,096,000$ & $1000^{* 1}=1,000$ \\
\hline $\begin{array}{l}\text { CL } \\
\text { Subtotal }\end{array}$ & $2,332,704$ & 1,376 \\
\hline $\begin{array}{l}\text { FCL } \\
\text { Subtotal }\end{array}$ & $58,621,952$ & 9,192 \\
\hline Total & $60,954,656$ & 10,568 \\
\hline
\end{tabular}

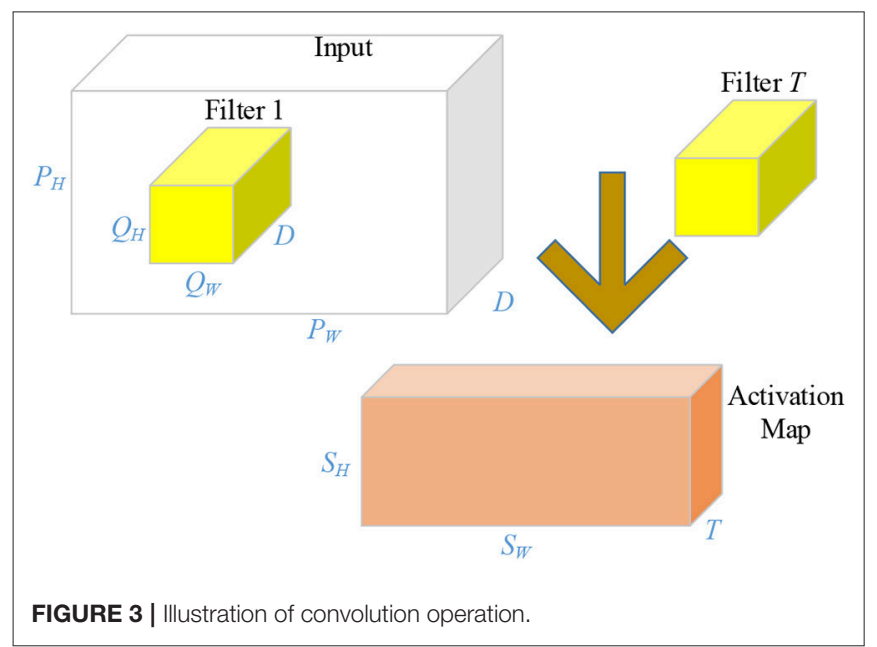

Fifth, the fully connected layers (FCLs) have connections to all activations in the previous layer, so they can be modeled as multiplying the input by a weight matrix and then adds a bias vector. The last fully-connected layer includes the equal number of artificial neurons as the number of total classes $C$. Therefore, each neuron in the last FCL represents the score of that cognate class, as shown in Figure 5.

Sixth, the softmax layer (SL), utilizes the multiclass generalization of logistic regression (32), also known as softmax function. SL is commonly connected after the final FCL. From the perspective of the activation function, the sigmoid/ReLU function works on a single input single output mode, while the SL serves as a multiple input multiple output mode, as shown in Figure 6. A toy example can be imagined. Suppose we have a four input at the final SL layer with values of (1-4), then after a softmax layer, we have an output of [0.032, 0.087, 0.236, 0.643].

Suppose that $T(f)$ symbolizes the prior class probability of class $f$, and $T(h \mid f)$ means the conditional probability of sample $h$ given class $f$. Then we can conclude that the likelihood of sample $h$ belonging to class $f$ is

$$
T(f \mid h)=\frac{T(h \mid f) \times T(f)}{\sum_{i=1}^{F} T(h \mid i) \times T(i)}
$$

Here $F$ stands for the whole number of classes. Let $\Omega_{f}$ equals

$$
\Omega_{f}=\ln [T(h, f) \times T(f)]
$$

Afterwards, we get

$$
T(f \mid h)=\frac{\exp \left(\Omega_{f}(h)\right)}{\sum_{i=1}^{F} \exp \left(\Omega_{i}(h)\right)}
$$

Finally, a dropout technique is used, since training a big neural network is too expensive. Dropout freezes neurons at random with a dropout probability $\left(P_{D}\right)$ of 0.5 . During training phase, those dropped out neurons are not engaged in both a forward and backward pass. During the test phase, all neurons are used but with outputs multiplied by $P_{D}$ of 0.5 (33).

It can be regarded as taking a geometric mean of predictive distributions, generated by exponentially-many smallsize dropout neural networks. Figure 7A shows a plain neural network with numbers of neurons at each layer as $(2,4,8,10)$, 


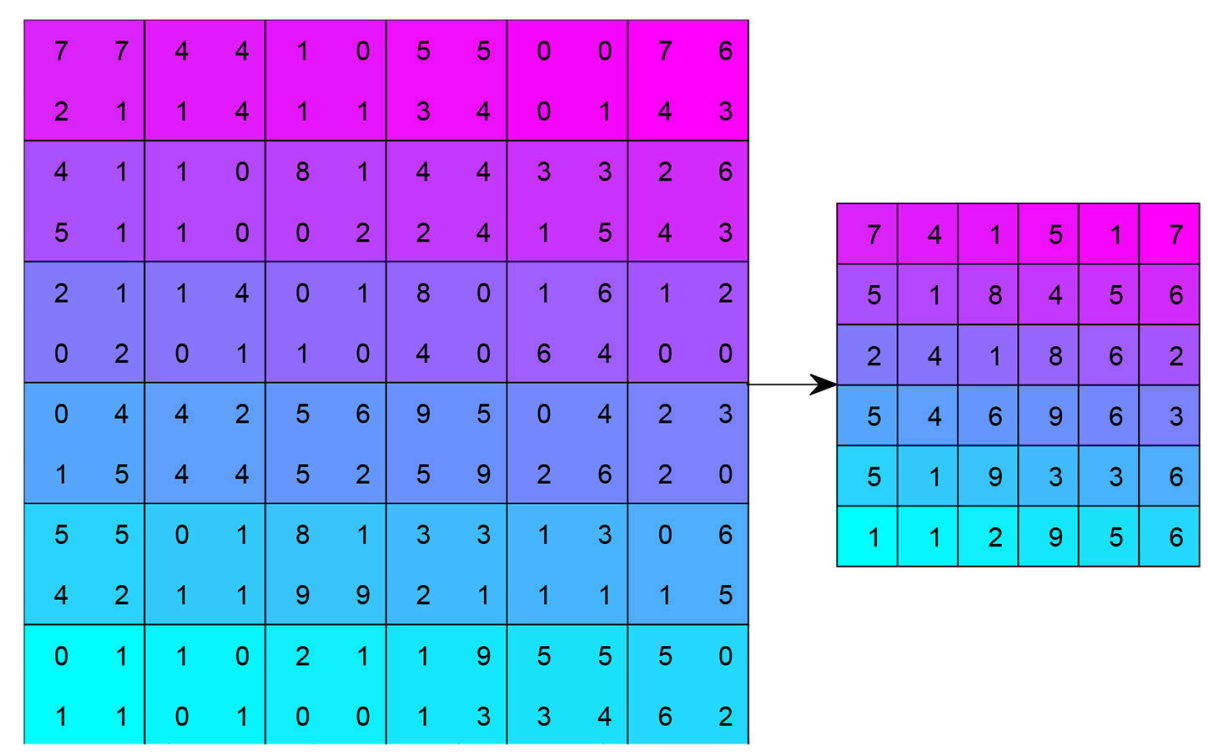

FIGURE 4 | Example of max pooling (stride =2, kernels size =2).

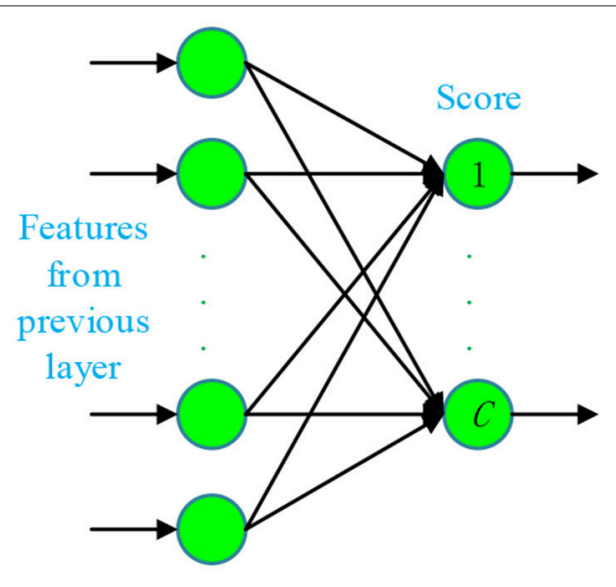

FIGURE 5 | Structure of last fully-connected layer ( $C$ stands for the number of total classes).

and Figure $7 \mathbf{B}$ shows the corresponding dropout neural network with $P_{D}$ of 0.5 , where only $(1,2,4,5)$ neurons remain active at each layer.

\section{Transfer AlexNet to Alcoholism Identification}

First, we needed to modify the structure. The last FCL was revised, since the original FCLs were developed to classify 1,000 categories. Twenty randomly selected classes were listed as: scale, barber chair, lorikeet, miniature poodle, Maltese dog, tabby, beer bottle, desktop computer, bow tie, trombone, crash helmet, cucumber, mailbox, pomegranate, Appenzeller, muzzle, snow leopard, mountain bike, padlock, diamondback. We observed that none of them are related to the brain image. Hence, we could not directly apply AlexNet as the feature extractor. Therefore, fine-tuning was necessary.

Since the length of output neurons in orthodox AlexNet (1000) is not equal to the number of classes in our task (2), we needed to revise the corresponding softmax layer and classification layer. The revision is shown in Table 4. In our transfer learning scheme, we used a new randomly-initialized fully connected layer with two neurons, a softmax layer, and a new classification layer with only two classes (alcoholism and non-alcoholism).

Next, we set the training options. Three subtleties were checked before training. First, the whole training epoch should be small for a transfer learning. In this study, we set the number of training epochs to 10 . Second, the global learning rate was set to a small value of $10^{-4}$ to slow learning down, since the early parts of this neural network were pre-trained. Third, the learning rate of new layers were 10 times that of the transferred layer, since the transferred layers with pre-trained weights/biases and new layers were with random-initialized weights/biases.

Third, we varied the numbers of transferred layers and tested different settings. The AlexNet consists of five conv layers (CL1, CL2, CL3, CL4, and CL5) and three fully-connected layers (FCL6, FL7, FL8). As a result, we tested five different transfer learning settings as shown in Figure 8 in total, in all experiments. For example, here Setting A means that the layers from the first layer to layer $\mathrm{A}$ are transferred directly with learning rate as $10^{-4}$ $\times 1=10^{-4}$. The late layers from layer $\mathrm{A}$ to the last layer are randomly initialized with a learning rate of $10^{-4} \times 10=10^{-3}$.

\section{Implementation and Measure}

We ran the experiment many times. Each time, the trainingvalidation-test division was set at random again. The training procedure stopped when either the algorithm reached maximum 

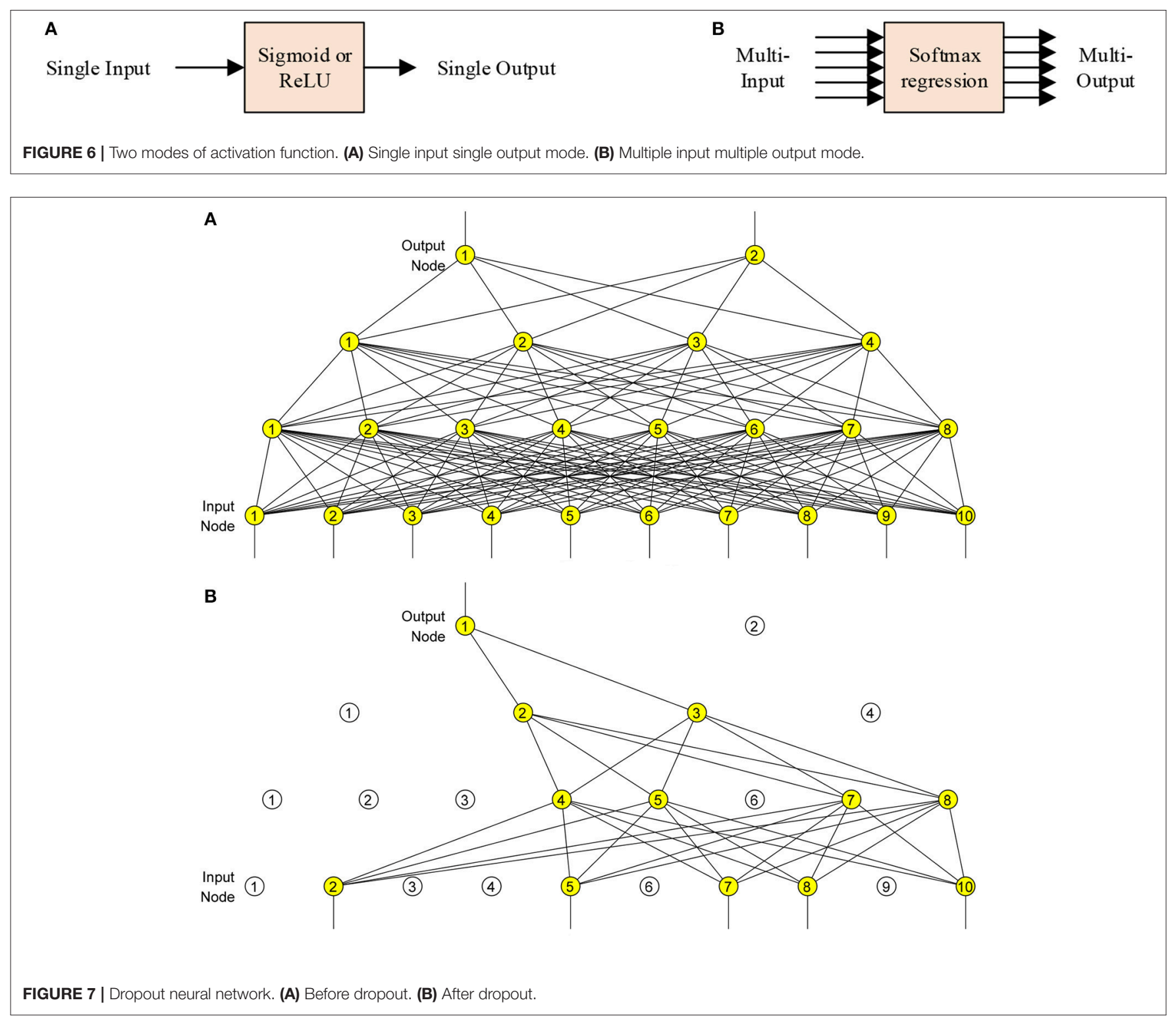

TABLE 4 | Revision of Last three layers of AlexNet.

\begin{tabular}{lll}
\hline Layer & Original & Replaced \\
\hline 23 & FCL (1000) with pre-trained & FCL (2) with random \\
& weights and biases & initialization \\
24 & Softmax Layer & Softmax Layer \\
25 & Classification Layer & Classification Layer \\
& (1,000 classes) & (two classes: alcoholism \\
& & and non-alcoholism) \\
\hline
\end{tabular}

epoch, or the performance of validation decreased over a preset training epoch. We repeatedly tuned the hyperparameters and found those optimal hyper-parameters based on a validation set. After the hyperparameters were fixed, we ran the final model on the test set for 10 runs. The test set confusion matrix across all runs was recorded, and the following five measures were calculated: sensitivity (SEN), specificity (SPC), precision (PRC), accuracy (ACC), and F1 score. Assume TP, $\mathrm{TN}, \mathrm{FP}$, and FN stands for true positive, true negative, false positive, and false negative, respectively, all five measures were defined as:

$$
\begin{array}{r}
S E N=\frac{T P}{T P+F N} \\
S P C=\frac{T N}{T N+F P} \\
P R C=\frac{T P}{T P+F P} \\
A C C=\frac{T P+T N}{T P+T N+F P+F N}
\end{array}
$$

$\mathrm{F}_{1}$ considers both the precision and the sensitivity to computer the score (34). That means, the measure of the "F1 score" is 


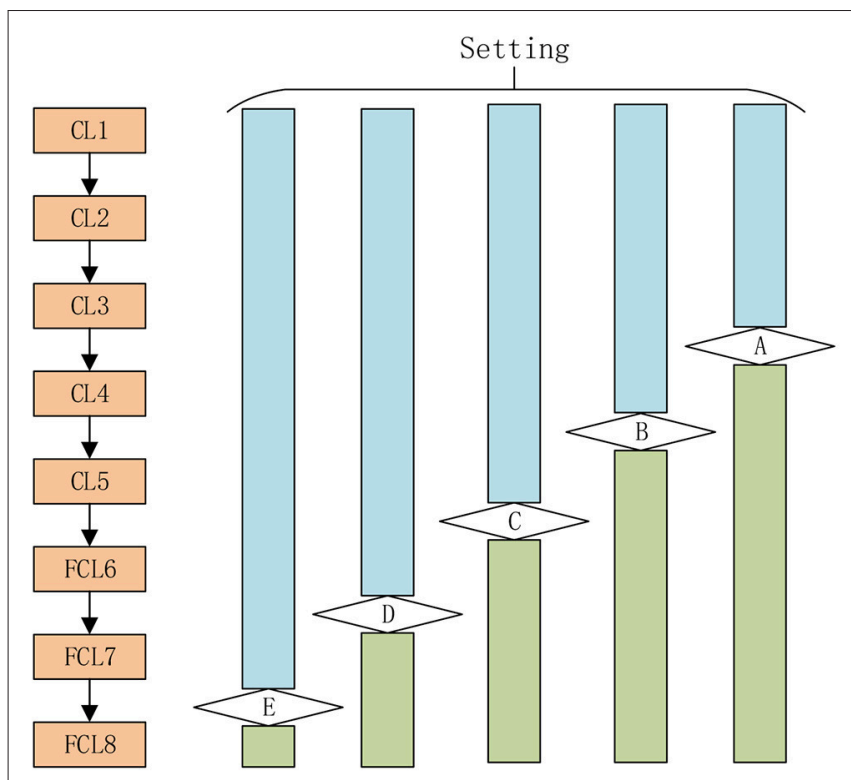

FIGURE 8 | Five different settings A-E (Setting A stands for the layers from first layer till layer $A$ are transferred layers, and the remaining layers are replaced layers).

the harmonic mean of the previous two measures: precision and sensitivity.

$$
F_{1}=\left(\frac{S E N^{-1}+P R C^{-1}}{2}\right)^{-1}
$$

Using simple mathematical knowledge, we can obtain:

$$
\begin{aligned}
F_{1} & =2 /\left(\frac{T P+F N}{T P}+\frac{T P+F P}{T P}\right) \\
& =2 /\left(\frac{2 T P+F P+F N}{T P}\right) \\
& =\frac{2 \times T P}{2 \times T P+F P+F N}
\end{aligned}
$$

Then, the average and standard deviation (SD) of all five measures of 10 runs of the test set were calculated and used for comparison. For ease of understanding, a pseudocode of our experiment is listed below in Table 5. The first block is to split the dataset into non-test and test sets. In the second block, the non-test set was split into training and validation randomly. The performance of the retrained AlexNet model was recorded and used to select the optimal transfer learning setting $\mathrm{S}^{*}$. In the final block, the performance on the test set via the retrained AlexNet using setting $\mathrm{S}^{*}$ was recorded and outputted.

\section{RESULTS}

\section{Data Augmentation Results}

Figure 9 shows the horizontally flipped image. Here, vertical flipping was not carried out because it can be seen as a combination of horizontal flipping with 180-degree rotation.
TABLE 5 | Pseudocode of our experiment.
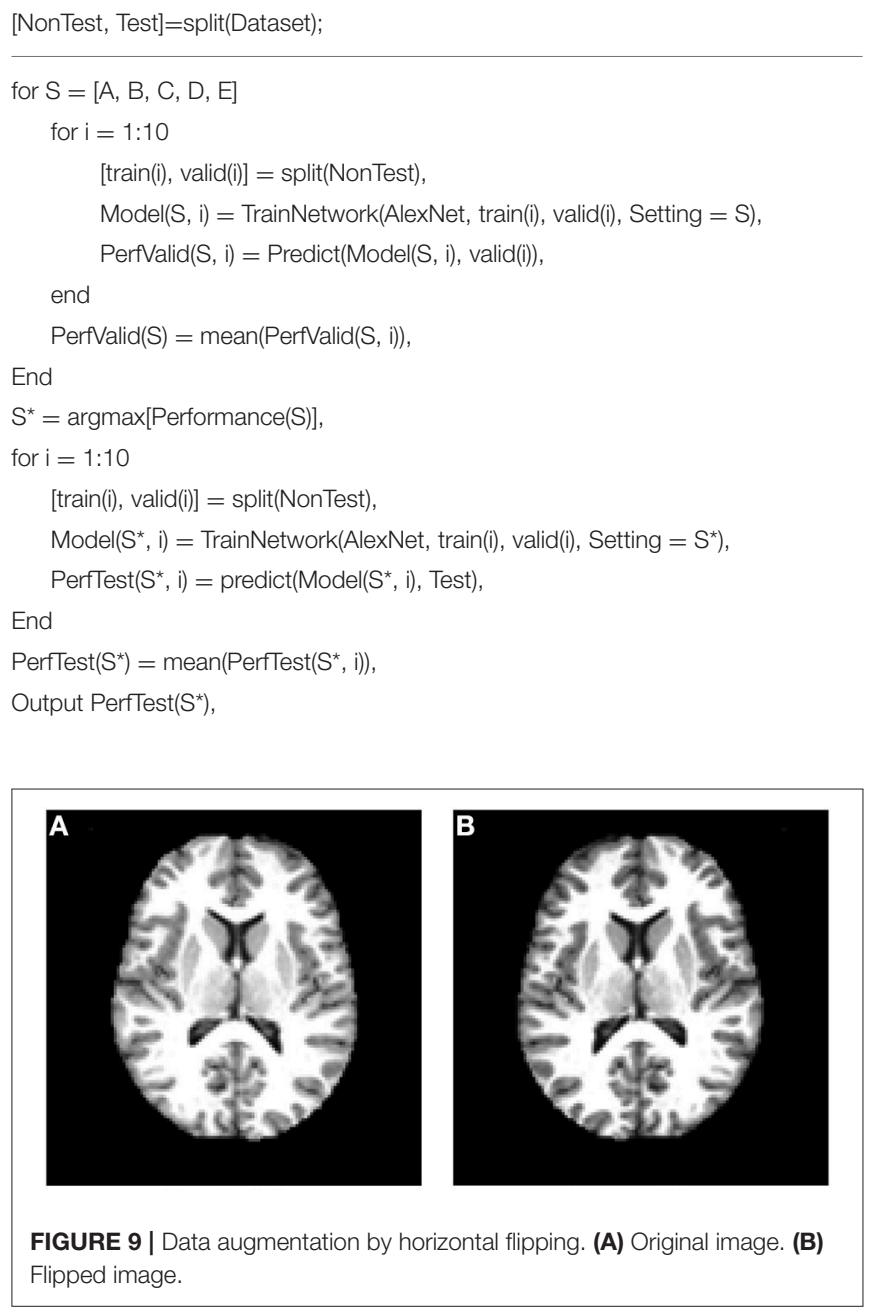

Figure 10 shows the data augmentation results of five different techniques: (a) noise injection; (b) scaling; (c) random translation; (d) image rotation; (e) Gamma correction. Due to the page limit, the data augmentation results on the flipped image are not shown.

\section{Comparison of Setting of TL}

In this experiment, we compared five different TL settings on the validation set. The results of Setting A are shown in Table 6, where the last row shows the mean and standard deviation value. The results of Setting $\mathrm{E}$ are shown in Table 7. Due to page limit, we only show the final results of Setting B, C, and $\mathrm{D}$ in Table 8.

Here, it can be seen from Table 8 that Setting E, i.e., replacing the FCL8, achieves the greatest performance among all five settings with respect to all measures. The reason may be (i) we expanded a relatively small dataset to a large training set using data augmentation; and (ii) the dissimilarity of our data and the original 1,000-category dataset. The first fact ensures that retraining avoids overfitting; and the latter fact 
TABLE 6 | Ten runs of validation performance of transfer learning using Setting A.

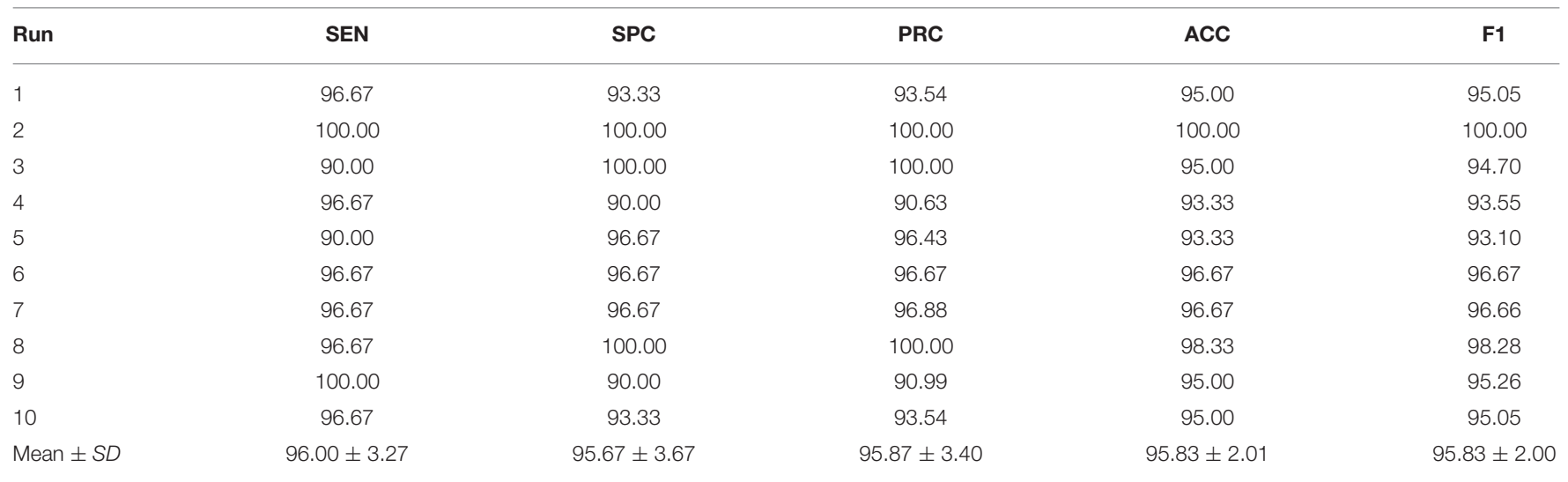

TABLE 7 | Ten runs of validation performance of transfer learning using Setting E.

\begin{tabular}{|c|c|c|c|c|c|}
\hline Run & SEN & SPC & PRC & ACC & F1 \\
\hline 1 & 93.33 & 100.00 & 100.00 & 96.67 & 96.55 \\
\hline 2 & 100.00 & 96.67 & 96.88 & 98.33 & 98.39 \\
\hline 4 & 100.00 & 100.00 & 100.00 & 100.00 & 100.00 \\
\hline 5 & 93.33 & 93.33 & 93.33 & 93.33 & 93.33 \\
\hline 8 & 96.67 & 93.33 & 93.54 & 95.00 & 95.05 \\
\hline 9 & 96.67 & 93.33 & 93.54 & 95.00 & 95.05 \\
\hline 10 & 100.00 & 100.00 & 100.00 & 100.00 & 100.00 \\
\hline Mean $\pm S D$ & $97.67 \pm 2.60$ & $97.67 \pm 3.00$ & $97.73 \pm 2.93$ & $97.67 \pm 2.38$ & $97.67 \pm 2.37$ \\
\hline A & $96.00 \pm 3.27$ & $95.67 \pm 3.67$ & $95.87 \pm 3.40$ & $95.83 \pm 2.01$ & $95.83 \pm 2.00$ \\
\hline B & $96.33 \pm 3.79$ & $96.00 \pm 2.49$ & $96.12 \pm 2.43$ & $96.17 \pm 2.36$ & $96.15 \pm 2.43$ \\
\hline C & $96.33 \pm 3.48$ & $96.33 \pm 3.14$ & $96.49 \pm 2.94$ & $96.33 \pm 2.08$ & $96.33 \pm 2.11$ \\
\hline D & $97.00 \pm 3.79$ & $97.00 \pm 2.77$ & $97.06 \pm 2.70$ & $97.00 \pm 2.56$ & $96.98 \pm 2.62$ \\
\hline E & $97.67 \pm 2.60$ & $97.67 \pm 3.00$ & $97.73 \pm 2.93$ & $97.67 \pm 2.38$ & $97.67 \pm 2.37$ \\
\hline
\end{tabular}

Bold means the best.

suggests that it is more practical to put most of the layers initialized with weights from a pretrained model, than freezing those layers. For clarity, we plotted the error bar and show it in Figure 11.

\section{Analysis of Optimized TL Setting}

The structure of the optimal transfer learning model (Setting E) is listed in Table 9. Compared to the traditional AlexNet model, the weights and biases of FCL8 were reduced from $4,096,000$ to 8,192 , and from 1,000 to 2, respectively. The main reason is that we only had two categories in our classification task. Thus, the whole weight of the deep neural network reduced slightly from 60,954,656 to $56,866,848$.
Nevertheless, we can observe that FCL6 and FCL7 still constitutes too many weights and biases. For example, FCL6 occupied $37,748,736 / 56,866,848=66.38 \%$ of the total weights in this optimal model, and FCL7 occupied $16,777,216 / 56,866,848=29.50 \%$ of the total weights. Additionally, the FCL subtotal comprised $95.90 \%$ of the total weights. This is the main limitation of our method. To solve it, we need to replace the fully connected layers with $1 \times 1$ conv layers. Another solution is to choose smallsize transfer learning models, such as SqueezeNet, ResNet, GoogleNet, etc.

\section{Effect of Data Augmentation}

This experiment compared the performance of runs with data augmentation against runs without data augmentation 
(DA). Configuration of transfer learning was set to Setting E. All the other parameters and network structures were the same as the previous experiments. The performance of the 10 runs without using DA are shown in Table 10. The results in terms of all measures are equal to or slightly above $95 \%$.

The comparison of using DA against not using DA is shown in Table 11. We can discern that DA indeed enhances the classification performance. The reason is that having a large dataset is crucial for good performance. The alcoholism image dataset is commonly of small size, and its size can be augmented to the order of tens of thousands (48,320 in this study). AlexNet can make full use of all its parameters with a big dataset. Without using DA, overfitting is likely to occur in the transferred model.

\section{Results of Proposed Method}

In this experiment, we chose Setting E (replace the final block) as shown in Figure 8. Here, the retrained neural network was tested on the test set. The results over all 10 runs on

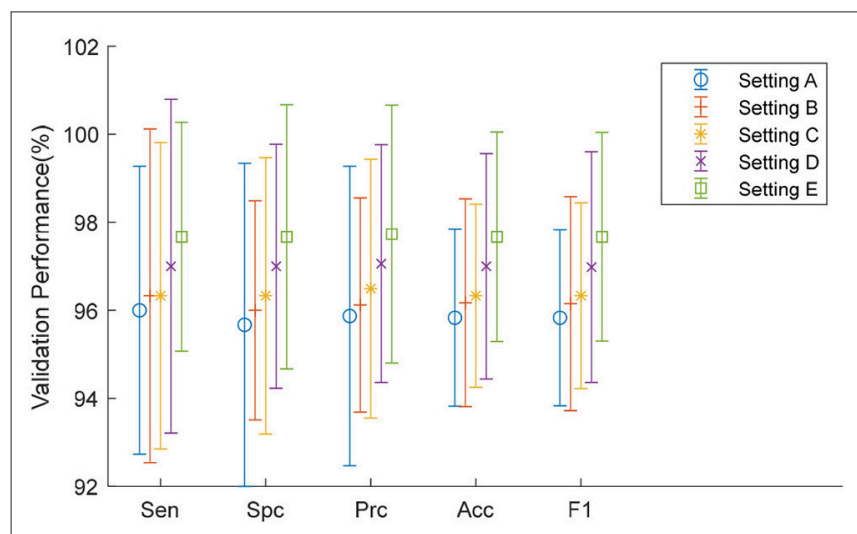

FIGURE 11 | Error bar of five TL settings. the test set are listed in Table 12 with details of sensitivity, specificity, precision, accuracy, and the F1 score of each run. Setting E yielded a sensitivity of $97.44 \pm 1.15 \%$, a specificity of $97.41 \pm 1.51 \%$, a precision of $97.34 \pm 1.49 \%$, an accuracy of $97.42 \pm 0.95 \%$, and an F1 score of $97.37 \%$ $\pm 0.97 \%$. Comparing Table 12 with Table 7, we can see that the mean value of test performance is slightly worse than that of the validation performance, but the standard deviation of the test performance is much smaller than that of the validation performance.

\section{Comparison to Alcoholism Identification Approaches}

This proposed transfer learning approach was compared with seven state-of-the-art approaches: PAC-PSO (4), HWT (5), LR (6), CSO (7), WRE (8), SVM-GA (9), and LMCoP (10). The comparison results are shown in Table 13. The cognate bar plot is shown in Figure 12. We can observe that our AlexNet transfer learning model has more than $3 \%$ improvement compared to the next best approach.

The reason is that this proposed model did not need to find features manually; nevertheless, it only used a learned feature from a pretrained model as initialization, and utilized the enhanced training set to fine-tune those learned features. This has two advantages: First, the development is quite fast, which can be reduced to $<1$ day. Second, the features can be fine-tuned to be more appropriate to this alcoholism classification task than other manually-designated features.

The bioinspired-algorithm may help retraining our AlexNet model. Particle swarm optimization (PSO) (35-37) and other methods will be tested. Cloud computing (38) in particular can be integrated into our method, to help diagnosis of remote patients.

TABLE 9 | Learnable layers in optimal transfer learning model.

\begin{tabular}{|c|c|c|c|c|}
\hline Name & Weights & Weights (\%) & Biases & Biases (\%) \\
\hline CL1 (Ours) & $11^{\star} 11^{*} 3^{*} 96=34,848$ & 0.06 & $1^{\star *} 1^{*} 96=96$ & 1.00 \\
\hline CL2 (Ours) & $5^{\star} 5^{\star} 48^{\star} 256=307,200$ & 0.54 & $1^{*} 1^{\star} 256=256$ & 2.68 \\
\hline CL4 (Ours) & $3^{*} 3^{\star} 192 * 384=663,552$ & 1.17 & $1^{*} 1^{*} 384=384$ & 4.01 \\
\hline CL5 (Ours) & $3^{\star} 3^{\star} 192^{\star} 256=442,368$ & 0.78 & $1^{*} 1 * 256=256$ & 2.68 \\
\hline FCL8 (AlexNet) & $1000 * 4096=4,096,000$ & & $1000 * 1=1,000$ & \\
\hline FCL8 (Ours) & $2^{*} 4096=8,192$ & 0.01 & $2^{*} 1=2$ & 0.02 \\
\hline CL Subtotal (AlexNet) & $2,332,704$ & & 1,376 & \\
\hline CL Subtotal (Ours) & $2,332,704$ & 4.10 & 1,376 & 14.38 \\
\hline FCL Subtotal (AlexNet) & $58,621,952$ & & 9,192 & \\
\hline
\end{tabular}


TABLE 10 | Ten runs without using data augmentation (Setting E).

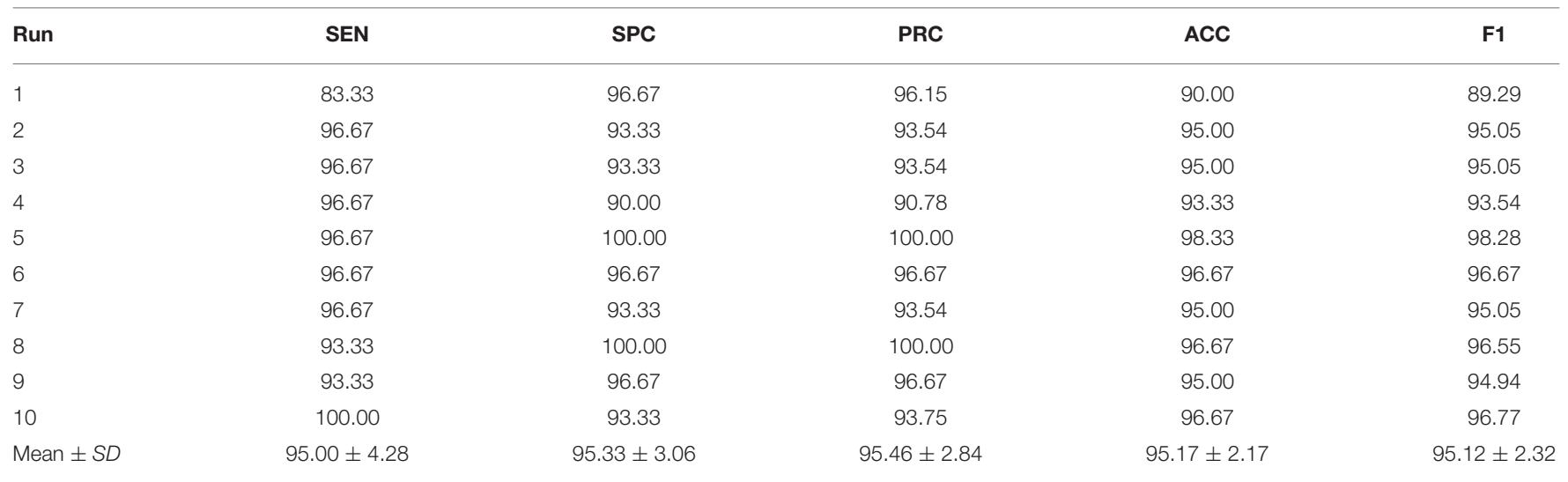

TABLE 11 | Effect of using data augmentation technique.

\begin{tabular}{lcccr}
\hline DA & SEN & SPC & PRC & ACC \\
\hline Not use DA & $95.00 \pm 4.28$ & $95.33 \pm 3.06$ & $95.46 \pm 2.84$ & $95.17 \pm 2.17$ \\
Use DA & $97.67 \pm 2.60$ & $97.67 \pm 3.00$ & $97.73 \pm 2.93$ & $97.67 \pm 2.38$ \\
(ours) & & & & $95.12 \pm 2.32$ \\
\end{tabular}

TABLE 12 | Ten runs of proposed method on the test set (Setting E).

\begin{tabular}{|c|c|c|c|c|c|}
\hline Run & SEN & SPC & PRC & $\mathrm{ACC}$ & F1 \\
\hline 1 & 97.44 & 96.31 & 96.22 & 96.86 & 96.82 \\
\hline 2 & 98.72 & 93.81 & 93.93 & 96.23 & 96.25 \\
\hline 4 & 97.44 & 98.75 & 98.72 & 98.11 & 98.07 \\
\hline 5 & 98.72 & 98.75 & 98.72 & 98.73 & 98.72 \\
\hline 8 & 97.44 & 98.75 & 98.75 & 98.12 & 98.05 \\
\hline 9 & 96.15 & 97.53 & 97.40 & 96.84 & 96.74 \\
\hline 10 & 97.44 & 97.53 & 97.44 & 97.48 & 97.44 \\
\hline Mean $\pm S D$ & $97.44 \pm 1.15$ & $97.41 \pm 1.51$ & $97.34 \pm 1.49$ & $97.42 \pm 0.95$ & $97.37 \pm 0.97$ \\
\hline
\end{tabular}

TABLE 13 | Comparison with state-of-the-art approaches.

\begin{tabular}{lccccc}
\hline Approach & SEN & SPC & PRC & ACC & F1 \\
\hline PAC-PSO (4) & 90.67 & 91.33 & 91.28 & 91.00 & 90.97 \\
HWT (5) & 81.71 & 81.43 & 81.48 & 81.57 & 81.60 \\
LR (6) & 84.00 & 84.86 & 84.73 & 84.43 & 84.36 \\
CSO (7) & 91.84 & 92.40 & 91.92 & 92.13 & 91.88 \\
WRE (8) & 93.60 & 93.72 & 93.35 & 93.66 & 93.47 \\
SVM-GA (9) & 88.42 & 88.93 & 88.27 & 88.68 & 88.34 \\
LMCoP (10) & 89.04 & 90.00 & 89.35 & 89.53 & 89.19 \\
AlexNet (Ours) & 97.44 & 97.41 & 97.34 & 97.42 & 97.37 \\
\hline
\end{tabular}

\section{CONCLUSIONS}

In this study, we proposed an AlexNet-based transfer learning method and applied it to the alcoholism identification task. This paper may be the first paper using transfer learning in the field of alcoholism identification. The results showed that this proposed approach achieved promising results with a sensitivity of $97.44 \pm 1.15 \%$, a specificity of $97.41 \pm 1.51 \%$, a precision of $97.34 \pm$ $1.49 \%$, an accuracy of $97.42 \pm 0.95 \%$, and an F1 score of $97.37 \pm 0.97$.

Future studies may include the following points: (i) other deeper transfer learning models, such as ResNet, DenseNet, GoogleNet, SqueezeNet, etc. should be tested; (ii) other data augmentation techniques should be attempted. Currently our dataset is small, so data augmentation may have a distinct effect on improving the performance; (iii) how to set the learning rate factor of each individual layer in the whole neural network, remains a challenge and needs to be solved; (iv) this method is ready to run on a larger dataset and can assist radiologists in their routine screening of brain MR images. 


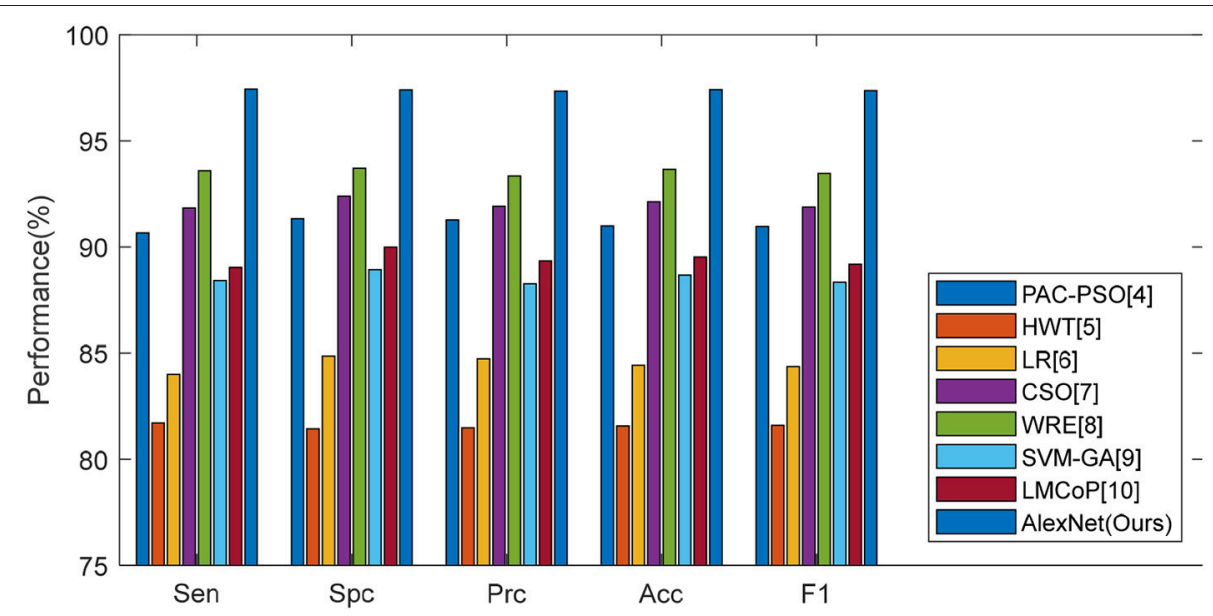

FIGURE 12 | Bar plot of comparison of eight algorithms.

\section{DATA AVAILABILITY}

The datasets for this manuscript are not publicly available because we need approval from our affiliations. Requests to access the datasets should be directed to yudongzhang@ieee.org.

\section{AUTHOR CONTRIBUTIONS}

S-HW and Y-DZ conceived the study. SX and XC designed the model. CT, JS, and DG analyzed the data. S-HW, XC, and Y$\mathrm{DZ}$ acquired the preprocessed data. SX and CT wrote the draft. S-HW, CT, JS, and Y-DZ interpreted the results. DG provided

\section{REFERENCES}

1. Khaderi SA. Alcohol and alcoholism introduction. Clinics in Liver Disease. (2019) 23:1-2. doi: 10.1016/j.cld.2018.09.009

2. Bilevicius E, Single A, Rapinda KK, Bristow LA, Keough MT. Frequent solitary drinking mediates the associations between negative affect and harmful drinking in emerging adults. Addict Behav. (2018) 87:115-21. doi: 10.1016/j.addbeh.2018.06.026

3. González-Reimers E, Romero-Acevedo L, Espelosín-Ortega E, MartínGonzález MC, Quintero-Platt G, Abreu-González P, et al. Soluble klotho and brain atrophy in alcoholism. Alcohol and Alcohol. (2018) 53:503-10. doi: 10.1093/alcalc/agy037

4. Hou XX. Alcoholism detection by medical robots based on $\mathrm{Hu}$ moment invariants and predator-prey adaptive-inertia chaotic particle swarm optimization. Comput Electric Eng. (2017) 63:126-38. doi: 10.1016/j.compeleceng.2017.04.009

5. Lima $\mathrm{D}$. Alcoholism detection in magnetic resonance imaging by Haar wavelet transform and back propagation neural network. AIP Conf Proc. (2018) 1955: 040012. doi: 10.1063/1.5033676

6. Macdonald F. Alcoholism detection via wavelet energy and logistic regression. Adv Intell Syst Res. (2018) 148:164-8. doi: 10.2991/icitme-18. 2018.33

7. Qian P. Cat Swarm Optimization applied to alcohol use disorder identification. Multimedia Tools Appl. (2018) 77:22875-96. doi: $10.1007 /$ s11042-018-6003-8
English revision of this paper. All authors provided critical revision and consent for this submission.

\section{FUNDING}

The authors are grateful for the financial support of the Zhejiang Provincial Natural Science Foundation of China (LY17F010003, Y18F010018), the National key research and development plan (2017YFB1103202), the Open Fund of Guangxi Key Laboratory of Manufacturing System and Advanced Manufacturing Technology (17-259-05-011K), the Natural Science Foundation of China (61602250, U1711263, U1811264), and the Henan Key Research and Development Project (182102310629).

8. Han L, Identification of Alcoholism based on wavelet Renyi entropy and three-segment encoded Jaya algorithm. Complexity. (2018) 2018:3198184. doi: $10.1155 / 2018 / 3198184$

9. Chen, Y. Alcoholism detection by wavelet entropy and support vector machine trained by genetic algorithm. In: 27th IEEE International Conference on Robot and Human Interactive Communication (ROMAN). Nanjing: IEEE. (2018). p. 770-5.

10. Jenitta A, Samson Ravindran R. Image retrieval based on local mesh vector co-occurrence pattern for medical diagnosis from MRI brain images. J Med Syst. (2017) 41:157. doi: 10.1007/s10916-017-0799-Z

11. Thangthai A, Milner B, Taylor S. Synthesising visual speech using dynamic visemes and deep learning architectures. Comput Speech Lang. (2019) 55:101-19. doi: 10.1016/j.csl.2018.11.003

12. Das A, Acharya UR, Panda SS, Sabut S. Deep learning based liver cancer detection using watershed transform and Gaussian mixture model techniques. Cogn Syst Res. (2019) 54:165-75. doi: 10.1016/j.cogsys.2018.12.009

13. Talo M, Baloglu UB, Yildirim Ö, Acharya UR. Application of deep transfer learning for automated brain abnormality classification using MR images. Cogn Syst Res. (2019) 54:176-88.

14. Zeng N, Zhang H, Liu W, Liang J, Alsaadi FA. A switching delayed PSO optimized extreme learning machine for short-term load forecasting. Neurocomputing. (2017) 240:175-82. doi: 10.1016/j.neucom.2017.01.090

15. Zeng N, Zhang H, Li Y, Liang J, Dobaie AM. Denoising and deblurring gold immunochromatographic strip images via gradient projection algorithms. Neurocomputing. (2017) 247:165-72. doi: 10.1016/j.neucom.2017.03.056 
16. Zeng N, Wang Z, Zhang H, Liu W, Alsaadi FE. Deep belief networks for quantitative analysis of a gold immunochromatographic strip. Cogn Comput. (2016) 8:684-92. doi: 10.1007/s12559-016-9404-x

17. Zeng $\mathrm{N}$, Wang $Z$, Zhang $\mathrm{H}$. Inferring nonlinear lateral flow immunoassay state-space models via an unscented Kalman filter. Sci China Inform Sci. (2016) 59:112204. doi: 10.1007/s11432-016-0280-9

18. Zeng N, Wang Z, Zineddin B, Li Y, Du M, Xiao L. et al. Image-based quantitative analysis of gold immunochromatographic strip via cellular neural network approach. IEEE Trans Med Imaging. (2014) 33:1129-36. doi: 10.1109/TMI.2014.2305394

19. Wang SH, Lv YD, Sui Y, Liu S, Wang SJ, Zhang YD. Alcoholism detection by data augmentation and convolutional neural network with stochastic pooling. J Med Syst. (2018) 42:2. doi: 10.1007/s10916-01 7-0845-x

20. Sangaiah AK. Alcoholism identification via convolutional neural network based on parametric ReLU, dropout, and batch normalization. Neural Comput Appl. (2019). doi: 10.1007/s00521-018-3924-0

21. Fahimi F, Zhang Z, Goh WB, Lee TS, Ang KK, Guan C. Inter-subject transfer learning with an end-to-end deep convolutional neural network for EEG-based BCI. J Neural Eng. (2019) 16:026007. doi: 10.1088/174 $1-2552 /$ aaf3f6

22. Comert Z, Kocamaz AF. Fetal hypoxia detection based on deep convolutional neural network with transfer learning approach. In: Software Engineering and Algorithms in Intelligent Systems. Silhavy R, editor. Cham: Springer International Publishing Ag (2019) p. 239-48.

23. Hussain M, Bird JJ, Faria DR. A study on CNN transfer learning for image classification. In: Advances in Computational Intelligence Systems. Lotfi A, Bouchachia H, Gegov A, Langensiepen C, McGinnity M, editors. Cham: Springer International Publishing Ag (2019) p. 191-202.

24. Soudani A, Barhoumi W. An image-based segmentation recommender using crowdsourcing and transfer learning for skin lesion extraction. Exp Syst Appl. (2019) 118:400-10. doi: 10.1016/j.eswa.2018.10.029

25. Ouchi T, Tabuse M. Effectiveness of data augmentation in automatic summarization system. In: Sugisaka M, Jia Y, Ito T, Lee JJ, editors. International Conference on Artificial Life and Robotics (ICAROB). Oita: Alife Robotics Co., Ltd. (2019). p. 177-80.

26. Krizhevsky A, Sutskever I, Hinton GE. ImageNet classification with deep convolutional neural networks. Commun ACM. (2017) 60:84-90. doi: $10.1145 / 3065386$

27. Mishkin D, Sergievskiy N, Matas J. Systematic evaluation of convolution neural network advances on the Imagenet. Comput Vis Image Understand. (2017) 161:11-19. doi: 10.1016/j.cviu.2017.05.007

28. Serra E, Sharma A, Joaristi M, Korzh O. Unknown landscape identification with CNN transfer learning. In: IEEE/ACM International Conference on Advances in Social Networks Analysis and Mining. Barcelona: IEEE (2018). p. 813-20.
29. Kanuri SN, Navali SP, Ranganath SR, Pujari NV. Multi neural network model for product recognition and labelling. In: 7th International Conference on Computing, Communications and Informatics (ICACCI). Bangalore: IEEE (2018) p. 1837-42.

30. Deng J, Dong W, Socher R, Li LJ. ImageNet: a large-scale hierarchical image database. In: 2009 IEEE Conference on Computer Vision and Pattern Recognition. Miami, FL (2009). p. 248-55.

31. Godin F, Degrave J, Dambre J, De Neve W. Dual Rectified Linear Units (DReLUs): a replacement for tanh activation functions in quasi-recurrent neural networks. Patt Recogn Lett. (2018) 116:8-14. doi: 10.1016/j.patrec.2018.09.006

32. Tuske Z, Tahir MA, Schluter R, Ney H. Integrating gaussian mixtures into deep neural networks: softmax layer with hidden variables. In International Conference on Acoustics, Speech, and Signal Processing. Brisbane, QLD: IEEE (2015). p. 4285-9.

33. Poernomo A, Kang DK. Biased dropout and crossmap dropout: learning towards effective dropout regularization in convolutional neural network. Neural Netw. (2018) 104:60-7. doi: 10.1016/j.neunet.2018.03.016

34. AlBeladi AA, Muqaibel AH. Evaluating compressive sensing algorithms in through-the-wall radar via F1-score. Int J Signal Imaging Syst Eng. (2018) 11:164-71. doi: 10.1504/IJSISE.2018.093268

35. Zeng NY, Wang Z, Zhang H, Alsaadi FE. A novel switching delayed PSO algorithm for estimating unknown parameters of lateral flow immunoassay. Cogn Comput. (2016) 8:143-52. doi: 10.1007/s12559-016-9396-6

36. Zeng N, Zhang H, Chen Y, Chen B, Liu Y. Path planning for intelligent robot based on switching local evolutionary PSO algorithm. Assembly Autom. (2016) 36:120-6. doi: 10.1108/AA-10-2015-079

37. Zeng NY, You Y, Xie L, Zhang H, Ye L, Hong W, et al. A new imaged-based quantitative reader for the gold immunochromatographic assay. Optik. (2018) 152:92-9. doi: 10.1016/j.ijleo.2017.09.109

38. Aldossary M, Djemame K, Alzamil I, Kostopoulos A, Dimakis A, Agiatzidou E. Energy-aware cost prediction and pricing of virtual machines in cloud computing environments. Fut Gen Comput Syst. (2019) 93:442-59. doi: 10.1016/j.future.2018.10.027

Conflict of Interest Statement: The authors declare that the research was conducted in the absence of any commercial or financial relationships that could be construed as a potential conflict of interest.

Copyright (c) 2019 Wang, Xie, Chen, Guttery, Tang, Sun and Zhang. This is an open-access article distributed under the terms of the Creative Commons Attribution License (CC BY). The use, distribution or reproduction in other forums is permitted, provided the original author(s) and the copyright owner(s) are credited and that the original publication in this journal is cited, in accordance with accepted academic practice. No use, distribution or reproduction is permitted which does not comply with these terms. 